\title{
Light-scattering properties of a woven shade-screen material used for daylighting and solar heat-gain control
}

\author{
Jacob C. Jonsson, Eleanor S. Lee, and Mike Rubin \\ Lawrence Berkeley National Laboratory, 1 Cyclotron Road MS70A-2255, Berkeley, CA 94720, \\ U.S.A.
}

\begin{abstract}
Shade-screens are widely used in commercial buildings as a way to limit the amount of direct sunlight that can disturb people in the building. The shade screens also reduce the solar heat-gain through glazing the system. Modern energy and daylighting analysis software such as EnergyPlus and Radiance require complete scattering properties of the scattering materials in the system.

In this paper a shade screen used in the LBNL daylighting testbed is characterized using a photogoniometer and a normal angle of incidence integrating sphere. The data is used to create a complete bi-directional scattering distribution function (BSDF) that can be used in simulation programs.

The resulting BSDF is compared to a model BSDFs, both directly and by calculating the solar heat-gain coefficient for a dual pane system using Window 6.
\end{abstract}

Keywords: Shade screens, light scattering, ray-tracing, integrating sphere

\section{INTRODUCTION}

Shade cloth is typically woven from vinyl coated polyester yarns. The weave pattern, color, and density varies by manufacturer and are selected for a given building application by the degree of privacy, attenuation of sunlight, solar heat gain control, daylight, and outside view required as well as other practical requirements such as aesthetic appearance. Shade cloth can be used in a wide variety of applications from exterior awnings to interior roller shades, where in the latter case the fabric is mounted to a roller and manually or mechanically raised and lowered. Interior roller shades are commonly used due to their inherent simplicity, low cost, and uncluttered aesthetic appeal.

Given the prevalence and wide spread use of shade cloth in buildings, it may be surprising to note that no accurate characterization of these outwardly appearing simple materials has been made in a comprehensive standardized manner. Solar optical data for all transparent specular glass currently manufactured world wide are available via the International Glazing Database (IGDB ${ }^{1}$ ). No such database is available for common but optically-complex systems such as roller shades, Venetian blinds, screens (i.e. for insects), etc. These simple systems are increasingly being used to improve building energy efficiency through automated motorized control ${ }^{2}$ and improved fabric design. With today's urgent need for innovative technologies that can reduce building energy use, peak demand, and carbon emissions comes the need for more accurate characterization of materials.

At present, building energy simulation software such as DOE-2 or EQuest (a derivative of DOE-2) rely on simple factors to estimate the thermal and daylighting impacts of shading systems. These simple factors can be obtained from published tables; for example, ASHRAE provides a limited set of attenuation coefficients, ${ }^{3}$ derived from limited measured data, to estimate the reduction in solar heat flux through unshaded glazing due to shading systems. More sophisticated simulation programs such as Radiance, ${ }^{4}$ EnergyPlus, ${ }^{5}$ and BC/LC ${ }^{6}$ require more extensive data not yet commonly available. EnergyPlus requires solar and visible transmittance and reflectance data at normal incidence to model shades and blinds with the assumption that the materials are perfectly diffusing. Radiance accepts material data and/or geometrical descriptions to determine daylighting impacts of

Further author information: (Send correspondence to J.C.J.) J.C.J.: E-mail: JCJonsson@lbl.gov, Telephone: +15104867329 
optically complex systems. Next-generation versions of these two programs are currently being developed to accept more complete scattering property data sets.

Today there are no standards how to rate light-scattering products for these purposes. This lack of methods is locked in a stalemate between input data not being produced and the fact that building simulation programs does not handle light scattering data. The future looks promising with the advances made in the simulation programs mentioned above. The need for complete scattering distributions must be observed by the measurement community and full data sets produced.

The parameter to use for describing the scattering properties is the Bi-directional Reflectance/Transmittance Distribution Function (BR/TDF) that was originally defined by Nicodemus. ${ }^{7}$ For samples with both reflecting and transmitting properties it is possible to use the term Bi-directional Scattering Distribution Function (BSDF) to simplify talking about it. This parameter describes the relation between the incident irradiance and outgoing radiance in a specific solid angle. In that sense it is only slightly different from the traditional concepts of reflectance and transmittance, the difference being that you need to define the solid angle of the outgoing scattered light. A BSDF value is commonly described as dependent on incident and outgoing angle (two pairs of spherical angles), but in reality the value generally depends on wavelength and polarization just like the classical parameters reflectance and transmittance.

At first glance it would seem that incomplete data sets could be used to simulate a lot of cases, e.g. only a limited number of incident angles are needed for direct sunlight incident on a window. The need for full BSDF data sets for materials becomes apparent when you start to do the multiple reflection between a diffusing material and other layers. Then information about all incident and outgoing angles will be needed. Tools for calculating such properties has previously not been available and any simulation would have to be through raytracing any multi-layer system which had a scattering layer in it. This is unpractical and would require the raytracer to have the scattering probability function of the scattering layer, information that is non-trivial to obtain.

The characterization problems included in studying scattering samples such as shade screens are many, from design compromises in the instruments to lack of standard procedures and reference characterization methods. The integrating sphere is designed only for direct-hemispherical outgoing data and commonly also only for normal angle of incidence. It is possible to obtain BTDF data for homogeneous samples using an integrating sphere, ${ }^{8}$ but the common method is to use a goniometer type instrument that has a detector scanning the outgoing hemisphere and measuring the angle-resolved scattering. The only organized inter-laboratory comparison of goniometer type instruments ${ }^{9}$ showed that different instruments are not always in agreement, sometimes widely disagreeing. All these measurement problems and lack of comprehensive standard methods forces data acquisition to include a verbose description of how it was carried out.

Without the possibility to obtain a complete set of experimental data a model is needed to complete the information while using as much of the available data as possible. A good model also guarantees that energy is conserved and that no unphysical values are produced. The $\mathrm{ABg}$-model ${ }^{10}$ is a general empirical model to produce a fairly simple scattering distribution.

When finally all the pieces comes together it will be possible to get quantifiable results to determine how good or bad light-scattering products perform for daylighting and energy conservation.

\section{THEORY}

The approach in this paper to obtain the complete BSDF for a sample follows three steps:

1. Measurement of as much information as possible about the sample.

2. Fitting of a scattering model to the experimental results.

3. Generation of a full BSDF according to the scattering model. 


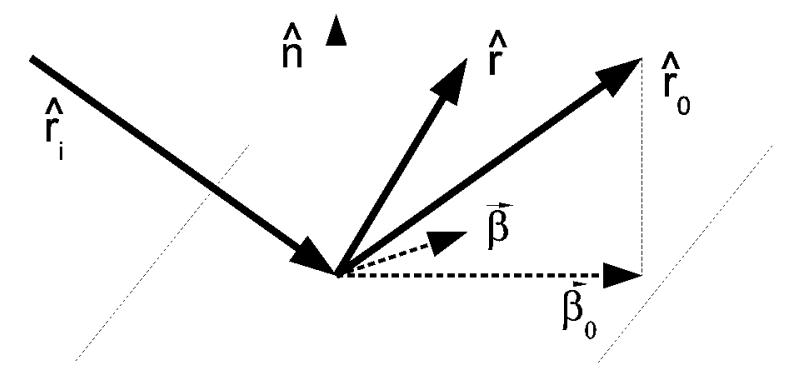

Figure 1. Definition of angular parameters used in the ABg-model. The direction vector for the incident light, $\hat{r}_{i}$, on a scattering surface with normal $\hat{n}$, is scattered in a direction $\hat{r}$ away from the specular direction $\hat{r_{0}}$. The projections on the surface of $\hat{r}$ and $\hat{r_{0}}$ are given by the vectors $\vec{\beta}$ and $\overrightarrow{\beta_{0}}$, respectively.

The first step is carried out a using goniophotometer and an integrating sphere spectrophotometer to obtain both angle-resolved scattering properties and direct-hemispherical information. Limitations created by the instruments and samples makes it impossible to obtain full data sets from experiments, typically data for retro-reflection directions as well as large angles of incidence is hard to obtain. The word full in this context denotes that scattering data are available for both the whole incident and outgoing hemisphere.

The second step makes use of the incomplete experimental data set to find parameters of a scattering model. This is done by minimizing the mean square error between the parametric model and the scattering data. Once the parameters have been obtained it is possible to calculate the full BSDF from the model.

\subsection{The ABg-model}

The ABg-model was presented by Freniere ${ }^{10}$ as a practical implementation of Harvey's concept of scattering transfer functions. ${ }^{11}$ It is a simple empirical scattering model with the advantage that it is a function of the difference between specular direction and the scattering direction. The function is given as

$$
\overline{B S D F}\left(\left|\beta-\beta_{0}\right|\right)=\frac{A}{B+\left|\vec{\beta}-\overrightarrow{\beta_{0}}\right|^{9}}
$$

where $A, B$, and $g$ are empirical parameters, and $\left|\vec{\beta}-\overrightarrow{\beta_{0}}\right|$ is defined from the scattering direction and specular direction as shown in Fig. 1. The angular term, $\left|\vec{\beta}-\overrightarrow{\beta_{0}}\right|$, is the scalar length of the difference between the scattering and specular direction vectors as projected on the scattering surface. It is 0 for the specular direction and has a theoretical maximum of 2 for total back-scattering at gracing incidence.

The parameters $A, B$, and $g$ do not correspond to any specific physical property, but each have a qualitative impact on the shape of the function that is noteworthy. The parameter $g$ controls the slope of the peak, making it sharper (i.e. more specular) with increasing $g$. For $g=0$ the resulting BSDF is Lambertian. The ratio $A / B$ defines the BSDF in the specular direction, i.e. $\left|\vec{\beta}-\vec{\beta}_{o}\right|=0$. The curve converges towards a derivative of zero as $\left|\vec{\beta}-\overrightarrow{\beta_{o}}\right|$ decreases, and for increasing $\left|\vec{\beta}-\overrightarrow{\beta_{o}}\right|$ converges to the power law $-g$. The parameter $B$ defines the roll-off point, i.e. the $\left|\vec{\beta}-\vec{\beta}_{0}\right|$ value where these two asymptotes cross. So, in essence, the model makes it possible to create distributions with a flat top that curves into a power-law slope.

During the work of this paper it was found beneficial to let the parameters $A, B$, and $g$ be dependent on angle of incidence. As noted in the previous paragraph, the BSDF in the specular direction is $A / B$ which is not true for all types of materials. Keeping $A / B$ constant results in a BSDF that is constant in the specular direction regardless of the angle of incidence.

\subsection{Measurement of BSDF data with a goniophotometer}

The angle-resolved data in this paper was acquired with an Optronics OL750-75MA instrument. The light source was a $150 \mathrm{~W}$ tungsten halogen lamp. The incident light was made monochromatic to a wavelength of $633 \mathrm{~nm}$ 


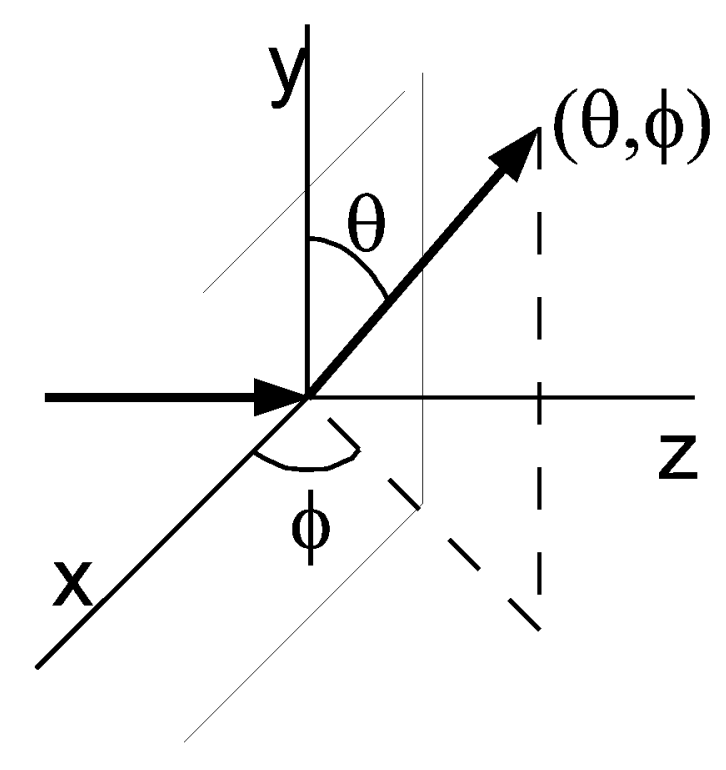

Figure 2. Definition of polar coordinates $\theta$ and $\phi$ for describing light being scattered from a sample in the $x y$-plane.

using a single grating monochromator with a blaze of $0.5 \mu \mathrm{m}$ and 1200 grooves $/ \mathrm{mm}$. The detector used was a $1 \mathrm{~cm}^{2}$ silicon detector mounted on an movable arm at a distance of $22 \mathrm{~cm}$ from the sample. The sample can be rotated to obtain different angles of incidence, and the detector arm moves in the plane of incidence that is created by the incident light and the sample normal.

The BSDF values for the given outgoing angle described by polar coordinates $\theta$ and $\phi$ can be obtained from the experiment according to the relation ${ }^{12}$

$$
B S D F(\theta, \phi)=\frac{r^{2}}{A_{\text {det }} \cos \theta} \frac{S_{\text {sample }}}{S_{\text {ref }}},
$$

where $r$ is the distance from the sample to the goniometer arm, $A_{\text {det }}$ the surface area of the detector, and $S_{\text {signal }}$ and $S_{r e f}$ are the measured signals for the sample and reference, respectively. The $S_{r e f}$ value is the signal obtained for the incident light and is measured in transmittance mode with no sample in place.

\subsection{Generating a complete BSDF}

The generation of a complete BSDF (BRDF and BTDF for light incident on both front and back surface) has been carried out in three steps. First the $\mathrm{ABg}$-model was fitted to the goniometer data, each angle of incidence was fitted separately to obtain multiple triplets of $\mathrm{A}, \mathrm{B}$, and $\mathrm{g}$. The trends seen in the acquired $\mathrm{A}$, B, and $\mathrm{g}$ were used in combination with the direct-hemispherical measurements to generate angle-dependent $\mathrm{A}$, B, and g-values for each sample and facing.

\section{EXPERIMENT AND SIMULATION}

\subsection{Material}

The shade screen material studied was Lutron's SheerShade basketweave 90 oyster/pearl gray model number SP14-90 with a 5\% openness. The threads are fiberglass coated with PVC. The material is fairly stiff compared to natural materials such as wool or cotton, but is still flexible. The thickness of the weave is $0.43 \mathrm{~mm}$ and the threads are paired to a width of $0.58 \mathrm{~mm}$. That the weave is thicker than the thickness of a single thread is due to the weaving between crossing threads and not surprising. The openings are not square but rather slightly rectangular. 


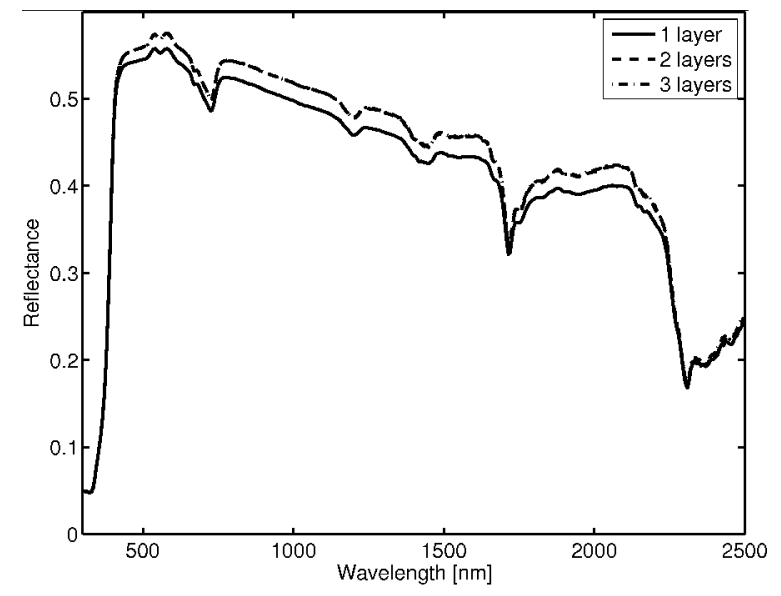

Figure 3. The reflectance of the shade screen for one, two, and three layers. The layers were positioned relative to each other to minimize the openness through the combination of the layers.

\subsection{Integrating sphere measurements}

The instrument used in this paper is a Perkin-Elmer Lambda 950 spectrophotometer fitted with a Labsphere $150 \mathrm{~mm}$ integrating sphere. This is a commercially available instrument that is commonly used for measuring transmittance and reflectance of scattering materials. However, there is no standard procedure for characterizing inhomogeneous materials like a shade screen.

\section{RESULTS}

\subsection{Integrating sphere measurements}

The shade screen model requires the thread reflectance. This is hard to obtain from the shade screen itself. One approximate method is to fold the screen so that the the second layer of screen fills the openness in the weave of the first layer.

If you disregard that multiple reflections from the second layer can be absorbed in the first the method should converge to the thread reflectance. Hence, this approximation is probably better for threads with higher reflectance, but the absolute error will be small for low reflectance threads so it might be acceptable there as well. We expect this approximation to be an estimate that is slightly lower than the true thread reflectance.

The reflectance of the Oyster Pearl Gray screen is shown for one, two, and three layers of the screen in Fig. 3. The measured values for two and three layers are identical. This is not surprising considering that the openness of the weave is $5 \%$ covering the gaps is easily done with just one extra layer. However, looking at the transmittance of the same number of layers, as shown in Fig. 4, we can notice that the transmittance does not go to zero with two layers. It is hard to explain why the transmittance does not converge to zero in the same way that the reflectance seems to converge to the final value. It is possible that the difference between how the sample is mounted resulted in the transmittance measurement being of a more loosely stacked layers, hence allowing more forward scattering to come through.

The diffuse only part of the transmittance was measured and the result is shown in Fig. 5 compared to the total transmittance. It is interesting to note that the undisturbed, specular part, is constant with regard to wavelength. This indicates that the threads are opaque in the entire wavelength region. This means that the diffuse transmittance is from forward scattering only. However, the transmittance increases with increasing wavelength while the reflectance is decreasing. This is surprising since one would expect that the forward scattering should be proportional to the reflectance of the opaque threads.

The openness of $5 \%$ given by the manufacturer is in close agreement with the measured specular transmittance. The slight difference might be due to the experimental definition of specular actually includes scattering up to 4 


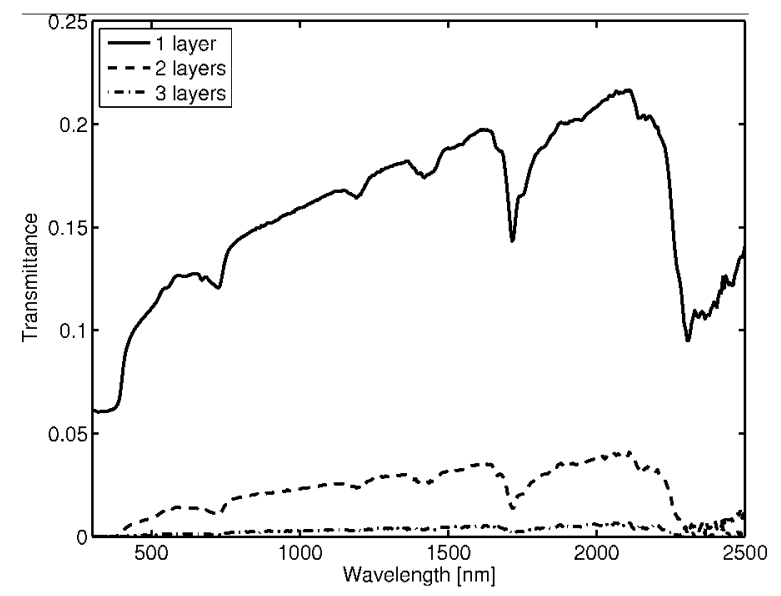

Figure 4. The transmittance of the shade screen for one, two, and three layers. The layers were positioned relative to each other to minimize the openness through the combination of the layers.

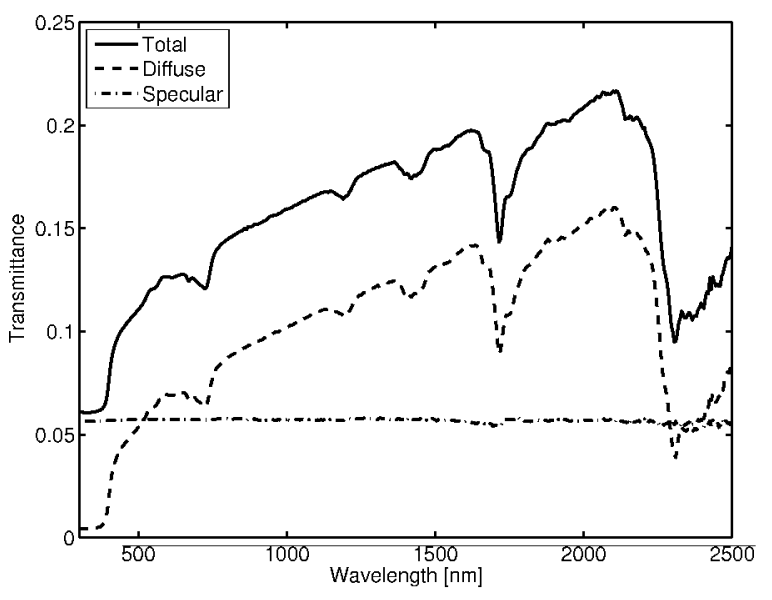

Figure 5. The measure total and diffuse only transmittance as a function of wavelength. The specular component is defined as the difference between the two measure components. 


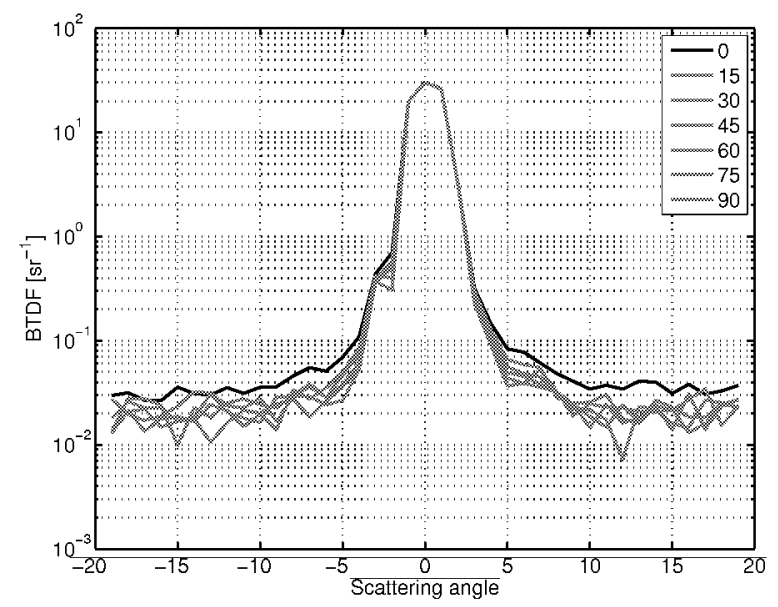

Figure 6. Angle-resolved scattering from the shade screen with the pattern of the weave rotated in different angles compared to the measurement plane.

a)

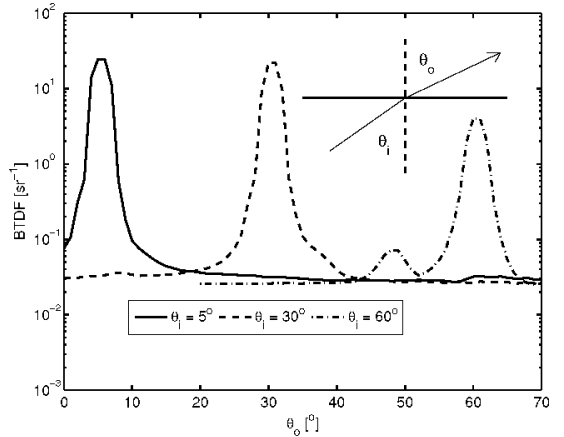

b)

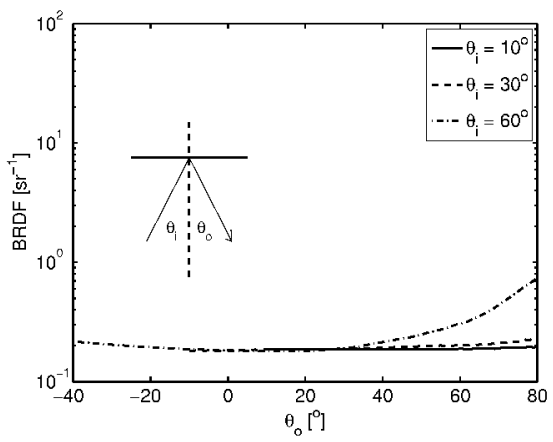

Figure 7. Measured BTDF and BRDF the three different angles of incidence. The inset shows the definition of incoming and outgoing angles with $0^{\circ}$ being equal to the sample normal.

degrees off the specular direction. This is a machine-dependent error and can therefore differ between different devices. It is obvious that the material is not opaque and maybe there is a part of that that is not scattered, this seems unlikely though considering the lack of wavelength dependence in the measured specular transmittance. Furthermore it is possible that the geometry of the light spot and the pattern of the weave resulted in a slight overrepresentation of openness. This error can be estimated by fixing the spectrophotometer to a single wavelength and scanning while moving sample in small increments so that the illuminated pattern varies. By recording maximum and minimum values obtained in this way it is possible to give the error of the measurement. For the studies sample the transmittance at $633 \mathrm{~nm}$ varied between 0.12 to 0.13 .

\subsection{Goniometer measurements}

Each sample was measured in 4 different configurations to obtain all possible combination of reflectance/transmittance and s-/p-polarization. The scattering measurements were only made for one plane of incidence, the geometrical contribution does not change the scattering much as shown in Fig. 6 . The measurements at the two polarization directions were averaged to a single value.

A few results are shown in Fig. 7 as example of how the experimental data looks. From Fig. 7a) it is clear that there is a strong specular contribution dominating the transmitted light. For $60^{\circ}$ angle of incidence there is also a second peak at lower angle than the specular direction. The reflectance is close to Lambertian as shown in Fig. 7b). The BRDF actually increases with increasing angle of incidence even though the intensity does not. This is possible since the threads does not actually constitute a flat surface, but rather is curved in a way that it will locally be normal to the incident light even as the angle of incidence is increased with respect to the 
a)
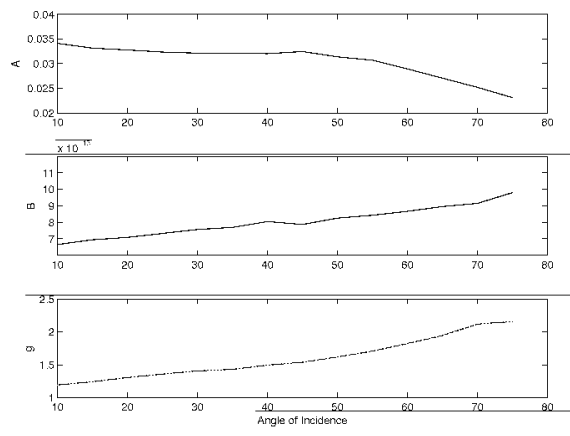

Figure 8. The fitting parameters as obtained for each measured angle of incidence. The transmittance fits are shown in a) and the reflectance fits in b). The sample in this graph is Oldcastle acid-etched. b)
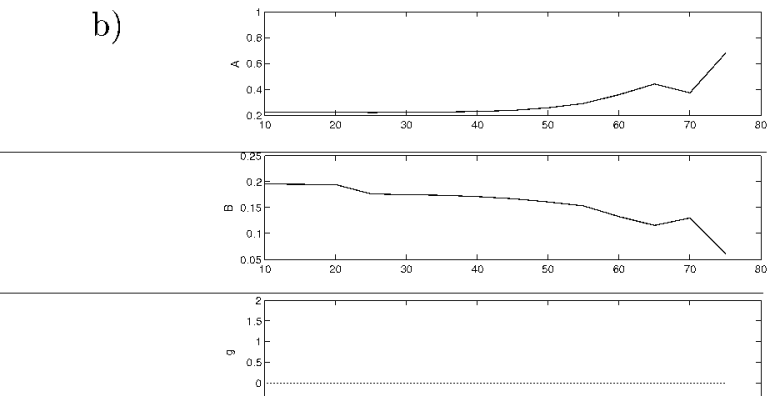

$\underset{\substack{40 \\ \text { Angle of Incidence }}}{50}$

macroscopic weave. That effect in combination with the definition of the BSDF from Eq. 2 with a cosine term in the denominator.

\subsubsection{Fitting of the $\mathrm{ABg}$ model}

All fitting was done with the standard simplex method implemented in the software MATLAB 7 from MathWorks, Inc. The error function between the fitted data and experimental data was a linear summed mean root square difference. Using a logarithmic error function gave less consistent results and was discarded, the balance between a few large BSDF values near $\left|\beta-\beta_{o}\right|=0$ and a lot of low values at larger $\left|\beta-\beta_{o}\right|$-values gave a good fit in linear error space. Furthermore, there was a high penalty for negative $A, B$, and g-values.

Looking at the results from fits for all angles of incidence, as shown in Fig. 8, may look discouraging at first as they seem to be far from constant and contains a few discontinuities. The exact reasons for the variation are not clearly determined, but the scattering distribution can not be independent of angle of incidence. This fact gets further support from the direct-hemispherical measurements which cannot be reproduced with a single $\mathrm{ABg}$ triplet. However, the amount of data obtained vary with angle of incidence, and data obtained at, or beyond, the limits of the instrument can affect the result. Without calibrated samples similar to the shade-screen samples, or other instruments to compare the result to, it is hard to determine the effect of each contribution.

When judging if the fit is good or bad one must ask how much relevance the different parts have. One thing to look at is the range in $\left|\beta-\beta_{o}\right|$ that a patch covers. At 40 degrees angle of incidence, the specular patch will include scattering in a range from $\left|\beta-\beta_{o}\right|<0.06$ (center to middle of side of the patch) to $\left|\beta-\beta_{o}\right|<0.11$ (center to corner of the patch). Add the nine nearest neighbors and you have all light scattered at $\left|\beta-\beta_{o}\right|<0.2$. Due to the lack of resolution in the Klems coordinate system in this range the actual shape is not of essence, however, the BSDF-value is large, so it can still have a noticeable impact on the direct-hemispherical value. For larger $\left|\beta-\beta_{o}\right|$-values the value is low, but there are a lot of patches. Therefore, the impact on the direct-hemispherical value is not insignificant for either part. As comparison one can consider a Lambertian distribution with light incident at normal angle of incidence, where more than $90 \%$ is scattered at $\left|\beta-\beta_{o}\right|$-values larger than 0.3 .

In the end, the graphs demonstrated in Fig. 8 was used with the direct-hemispherical results to generate ABg-parameters that depended on angle of incidence and had a correct direct-hemispherical value.

\subsubsection{Creation of the BSDF}

The BRDF and BTDF values were generated by creating a surface with the selected ABg-parameters in the raytracing program TracePro. The software is set up so that a sphere broken up into the patches defined by the Klems coordinate system is used as detector. An alternative, and maybe more obvious, way would be to analytically integrate the ABg-equation over each individual patch and find a constant value that corresponds to the same integrated value. However, the raytracing method was chosen since that will be convenient for window systems where the shade screen is combined with other elements. 
a)

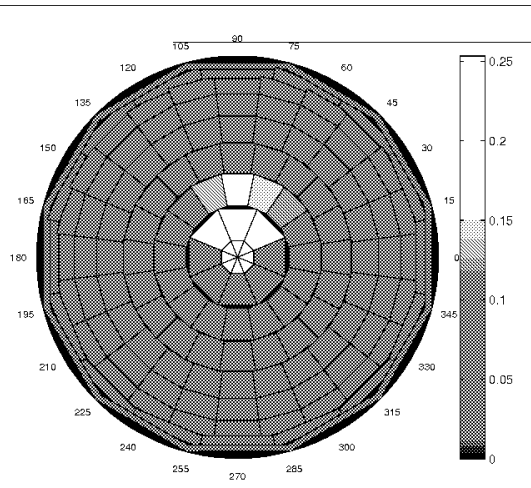

b)

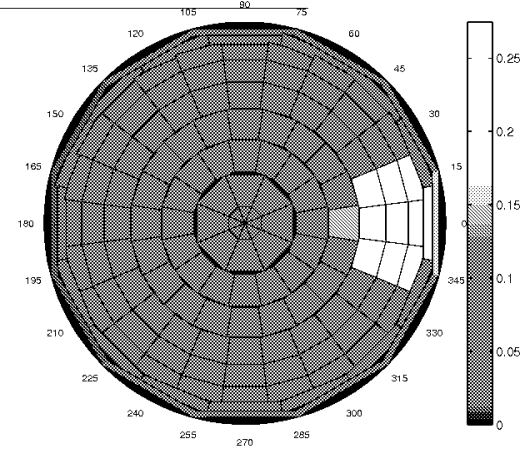

Figure 9. Virtual hemisphere projections of the BTDF values for the shade screen, in a) at incidence angles $\theta=10^{\circ}$ and $\phi=270^{\circ}$ and in b) at incidence angles $\theta=50^{\circ}$ and $\phi=180^{\circ}$. The unit is $s r^{-1}$.

a)

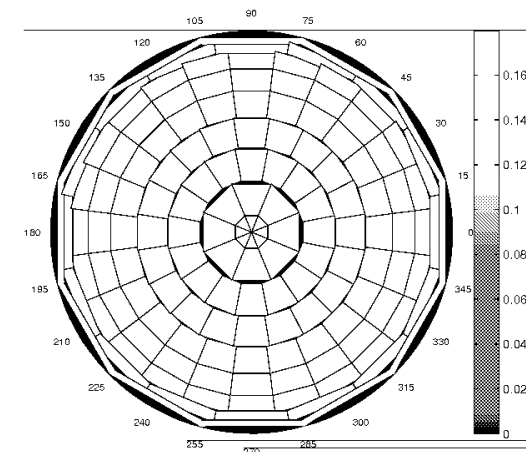

b)

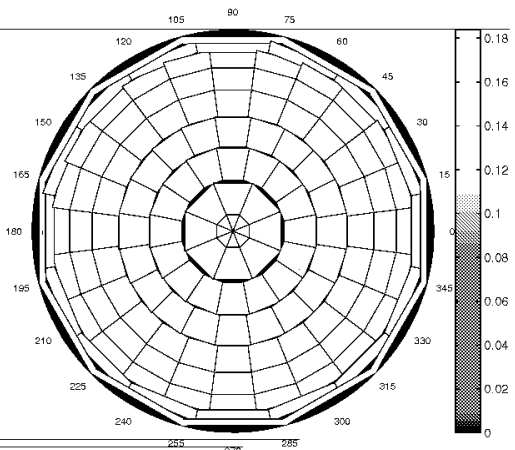

Figure 10. Virtual hemisphere projections of the BRDF values for the shade screen, in a) at incidence angles $\theta=10^{\circ}$ and $\phi=270^{\circ}$ and in b) at incidence angles $\theta=50^{\circ}$ and $\phi=180^{\circ}$. The unit is $s r^{-1}$.

Simplified methods using the value of the ABg-equation for the center of the patch, or even a mean of the center and the four corners, was found to result in significantly inaccurate, and sometimes unphysical, directhemispherical values, hence is not viable as a simpler alternative.

One way to visualize scattering distributions is through projection on a virtual hemisphere, this is done by dividing a virtual hemisphere into the patches defined by the coordinate system and attributing a false color corresponding to the BSDF-value of the patch. Figures 9-10 are examples of such representation of the data. The figures show how the symmetry of the scattering around the specular direction varies with sample and angle of incidence. It is interesting to notice the variation in shape and size of the nearest neighbor patches to the specular patch in the 10 degree angle of incidence case. This variation complicates any attempt to make a simpler model to determine the full scattering distribution from incomplete data.

\subsection{Simulation in Window 6}

A woven shade screen model has been incorporated in Window 6 , it is a geometrical radiosity model ${ }^{13}$ assuming that the threads are Lambertian and opaque. Furthermore the geometry is symmetrical and have a square pattern with constant spacing and thread thickness. The model is therefore not expected to agree with the studied sample but it is still relevant to see if it could be used.

The selected parameters for the model was a thread reflectance of 0.5 , a thread thickness of $0.58 \mathrm{~mm}$ and a center to center spacing of $0.686 \mathrm{~mm}$ to obtain a $5 \%$ geometrical openness.

The direct-hemispherical results versus angle of incidence from the model are presented in Fig. 11. These values does not look convincing in their simplicity. The transmitted component looks correct with regards to the undisturbed part which is expected to start at the openness and the decrease with increasing angle of incidence. 


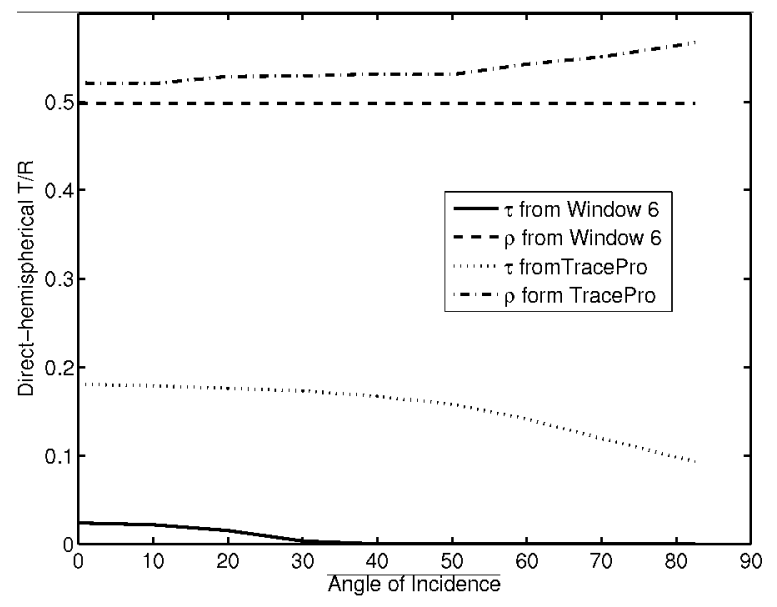

Figure 11. The direct-hemispherical transmittance, $\tau$, and reflectance, $\rho$, as function of angle of incidence according to the shade model implemented in Window 6 and from the complete BSDF generated with TracePro.

However, this decrease is expected to show up as an increase in the reflectance, but the reflectance is constant with respect to angle of incidence, and also higher than $95 \%$ of 0.5 which the given thread reflectance. It is therefore probable that there is a bug in the computations in Window 6.2.10.

\section{CONCLUSIONS}

An approximate method to obtain the thread reflectance was demonstrated with good results. This is needed when the thread material is not manufactured in larger pieces so that measurement of the bulk properties can be carried out.

A scattering distribution for all incident and outgoing angles was created based on the $\mathrm{ABg}$ model fitted to data obtained by a photogoniometer. This is a process that requires not commonly available equipment as well as significant time to do the fitting and analysis. A simpler model to obtain the BSDF based only on geometry and thread reflectance would be a significant help for simulation of these materials.

The radiosity model implemented in Window 6 did not agree with the experimental data. Careful examination of the generated BSDF data suggested that the scattered part was missing or too small. The source of this could not be determined but the authors of Window 6 are looking in to the issue and proper data is expected in the future.

The most puzzling part of the paper is the wavelength dependence of the reflectance and transmittance as measured with the integrating sphere. How can the diffuse transmittance increase with wavelength at the same time as the reflectance is decreasing?

\section{ACKNOWLEDGMENTS}

This work was supported by the Assistant Secretary for Energy Efficiency and Renewable Energy, Office of Building Technology, State, and Community Programs, of the U.S. Department of Energy under Contract No. DE-AC02-05CH11231.

\section{REFERENCES}

[1] Lawrence Berkeley National Laboratory, "IGDB - the Iinternational Glazing DataBase," (2007). http://windows.lbl.gov/materials/igdb/.

[2] Lee, E. S., Selkowitz, S. E., Hughes, G. D., Clear, R. D., Ward, G. J., Mardaljevic, J., Lai, J., Inanici, M., and Inkarojrit, V., "Daylighting the new york times headquarters building: Final report," Tech. Rep.

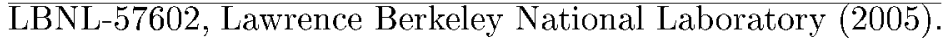


[3] Owen, M. S., ed., [2005 ASHRAE Handbook of Fundamentals], ASHRAE, Atlanta (2005).

[4] "Radiance." http://radsite.lbl.gov/radiance/HOME.html.

[5] "Energyplus." http://www.eere.energy.gov/buildings/energyplus/.

[6] Hviid, C. A., Nielsen, T. R., and Svendsen, S., "Simple tool to evaluate the impact of daylight on building energy consumption," Solar Energy (2008). doi:10.1016/j.solener.2008.03.001.

[7] Nicodemus, F. E., "Reflectance nomenclature and directional reflectance and emissivity," Applied Optics 9(6), 1474-1475 (1970).

[8] Jonsson, J. C. and Brandén, H., "Obtaining the bidirectional transmission distribution function of isotropically scattering materials using an integrating sphere," Optics Communications 277, 228-236 (2007).

[9] Platzer, W. J., "The ALTSET project: Measurement of angular properties for complex glazing," in [Proceedings of the Third International ISES Europe (Eurosun) Conference], (2000).

[10] Freniere, E. R., Gregory, G. G., and Chase, R. C., "Interactive software for optomechanical modeling," in [Proc. SPIE], Fisher, R., Johnson, B., and Juergens, R., eds., Stray Light Problems in Optical Systems 3130, 128 (1997).

[11] Harvey, J. E., "Light-scattering characteristics of optical surfaces," in [Proc. SPIE], Lytle, J. and Morrow, H., eds., Stray Light Problems in Optical Systems 107, 41-47 (1977).

[12] Earp, A., Smith, G., and Franklin, J., "Simplified BRDF of a non-Lambertian diffuse surface," Lighting Research Technology 39(3), 265-281 (2007).

[13] Curcija, C., "Implementation of wovenshade method in layeroptics.dll," (2006). http://windows.lbl.gov/software/window/6/Woven\%20Shade\%20Technical\%20Documentation.pdf. 